\title{
Limitations of the adiabatic approximation to the gravitational self-force
}

\author{
Adam Pound, Eric Poisson, and Bernhard G. Nickel \\ Department of Physics, University of Guelph, Guelph, Ontario, Canada N1G $2 W 1$
}

(Dated: November 28, 2005)

\begin{abstract}
A small body moving in the field of a much larger black hole and subjected to its own gravity moves on an accelerated world line in the background spacetime of the large black hole. The acceleration is produced by the body's gravitational self-force, which is constructed from the body's retarded gravitational field. The adiabatic approximation to the gravitational self-force is obtained instead from the half-retarded minus half-advanced field. It is much easier to compute, and it is known to produce the same dissipative effects as the true self-force. We argue that the adiabatic approximation is limited, because it discards important conservative terms which lead to the secular evolution of some orbital elements. We argue further that this secular evolution has measurable consequences; in particular, it affects the phasing of the orbit and the phasing of the associated gravitational wave. Our argument rests on a simple toy model involving a point electric charge moving slowly in the weak gravitational field of a central mass; the charge is also subjected to its electromagnetic self-force. In this simple context the true self-force is known explicitly and it can cleanly be separated into conservative and radiation-reaction pieces. Its long-term effect on the particle's orbital elements can be fully determined. In this model we observe that the conservative part of the self-force produces a secular regression of the orbit's periapsis. We explain how the conclusions reached on the basis of the toy model can be extended to the gravitational self-force, and we attempt to extend them also to the case of rapid motions and strong fields. While the limitations of the adiabatic approximation are quite severe in a post-Newtonian context in which the motion is slow and the gravitational field weak, they may be less severe for rapid motions and strong fields.
\end{abstract}

\section{INTRODUCTION}

The gravitational inspiral of a solar-mass compact object into a massive black hole residing in a galactic center has been identified as one of the most promising sources of gravitational waves for the Laser Interferometer Space Antenna [1]. The need for accurate theoretical models of the expected signal, for the purposes of signal detection and source identification, has motivated an intense effort from many workers to determine the motion of the small body in the field of the large black hole. This is done in a treatment that goes beyond the geodesic approximation and takes into account the body's own gravitational field, which is a small perturbation over the field of the black hole; this must be done without relying on slow-motion or weak-field approximations. In this treatment the small body can be thought of as moving on a geodesic of a perturbed spacetime, or equivalently, it can be thought of as moving on an accelerated world line in the background spacetime of the large black hole. The second point of view is generally adopted, and the body is said to move under the influence of its own gravitational selfforce [2, 3]. The self-force is derived from the retarded gravitational perturbation produced by the moving body. For a review of the self-force formalism, see Ref. [4].

The concrete evaluation of the gravitational self-force acting on a small body moving in the Kerr spacetime is a challenging project that has not yet been completed. Part of this challenge is concerned with the reconstruction of the metric perturbation [5, 6, 7] from the Teukolsky variables, which can be more practically evaluated [8]. Another is concerned with the regularization of the body's retarded field near the world line
9, 10, 11, 12, 13], in a context where it is expressed as an infinite sum over spherical-harmonic modes. Yet another challenge resides in the fact that the gravitational self-force is a gauge-dependent quantity [14]; this implies that the improved equations of motion must be incorporated in a self-consistent wave-generation formalism before they can yield gauge-invariant waveforms.

\section{ADIABATIC APPROXIMATION...}

Given this state of affairs, it is tempting to seek approximations to the self-force formalism that may bypass some of these challenges but still produce acceptably accurate results. One such approximation was formulated by Mino 15, 16, 17], who showed that the longterm evolution of the three principal orbital elements (the body's orbital energy, angular momentum, and Carter constant, which would all be constant under geodesic motion but instead evolve under self-forced motion) can be reproduced on the basis of a radiation-reaction force constructed from the half-retarded minus half-advanced solution to the perturbation equations. In particular, Mino showed that the total work done by the radiation-reaction force equals the total gravitational-wave energy radiated by the moving body, a result that was previously established by Quinn and Wald [18] and Gal'tsov [19]. Mino was further able to show that these long-term evolutions are, to a very good approximation, gauge-invariant.

Because the retarded and advanced fields are equally singular on the world line, their subtraction produces a regular field, and the computation of the radiationreaction force requires no regularization. This computation is therefore much simpler than the calculation of 
the true self-force, and the gauge-invariant nature of the radiation-reaction force argues in favor of its direct involvement in waveform calculations.

The approximation of the true self-force by the radiation-reaction force discards the rapid, oscillatory changes of the three principal orbital elements which occur on the time scale of the orbital period; it retains only the slow changes that survive after time-averaging. This replacement of the true self-force by the radiationreaction force in a calculation of the orbital evolution is called the adiabatic approximation. The computation of inspirals in the adiabatic approximation, and of their associated gravitational-wave signals, has so far been pursued by at least two research groups [20, 21, 22, 23].

Confidence in the reliability of the adiabatic approximation rests on the results of Mino (which are fully reliable) and the belief that all secular effects associated with the true self-force are embodied in the long-term evolution of the three principal orbital elements (energy, angular momentum, and Carter constant). Because the true self-force differs from the radiation-reaction force by purely conservative terms (which do no work), this is a statement of belief that the conservative part of the true self-force produces only short-term effects that do not accumulate over time and do not survive after time-averaging. This belief is expressed, for example, by Drasco, Flanagan, and Hughes [20], who state near the beginning of their Sec. 1.2: "The effect of the dissipative pieces of the self force will accumulate secularly, while the effect of the conservative pieces will not. Hence the effect of the dissipative pieces on the phase of the orbit will be larger than that of the conservative pieces by a factor of the number of cycles of inspiral."

\section{III. ... AND ITS LIMITATIONS}

Our purpose in this paper is to show that this belief is not well founded: The conservative terms in the true selfforce do produce effects that accumulate over time and significantly affect the phasing of the orbit. Our methods allow us to conclude that these effects are important in slow-motion, weak-field situations. They do not, however, allow us to investigate directly more interesting situations involving rapid motions and strong fields. Nevertheless, a tentative extrapolation of our slow-motion results indicate that these effects may be less important in strong-field situations.

While the radiation-reaction force captures the secular evolution of the three principal orbital elements (energy, angular momentum, and Carter constant), it makes no statement regarding the evolution of the remaining three orbital elements. (We shall call these, for reasons that will become clear presently, the positional orbital elements.) In particular, the radiation-reaction force does not capture the secular evolution of the positional elements.

In the case of geodesic motion all six orbital elements are constants of the motion. The three principal elements serve to label the geodesic, and the three positional elements serve to specify the body's initial position on this geodesic. Under orbital evolution driven by the true selfforce, all six elements vary with time, and the motion is tangent to an evolving osculating geodesic. While it is clear that the principal elements must evolve secularly, we intend to show that the positional elements also can evolve secularly, and that this evolution is driven by the conservative part of the true self-force. The adiabatic approximation, therefore, does not account for the secular evolution of the positional elements, and we intend to show that this leads to measurable phasing effects in the waves.

\section{ELECTROMAGNETIC SELF-FORCE IN A WEAKLY CURVED SPACETIME}

Our argument is based on a simple toy problem in which the true self-force can be evaluated explicitly and decomposed cleanly into conservative and radiationreaction pieces. The toy problem involves a point electric charge $q$ of mass $m$ moving slowly in the weak gravitational field of a (noncompact) star of mass $M$. In the toy problem the charge is a substitute for the small compact body, the electromagnetic radiation is a substitute for the gravitational radiation, and the star is a substitute for the massive black hole. We take the star to be immobile and we place it at the origin of a threedimensional Cartesian coordinate system $\boldsymbol{x}=[x, y, z]$. The electromagnetic self-force acting on the point charge was calculated by DeWitt and DeWitt [24], building on the foundations laid by DeWitt and Brehme 25]; this calculation was reviewed recently in Ref. 26]. Adopting a three-dimensional, Newtonian language throughout this section, the equations of motion are

$$
m \frac{d^{2} \boldsymbol{x}}{d t^{2}}=m \boldsymbol{g}+\boldsymbol{f}_{\text {self }},
$$

where $\boldsymbol{x}(t)$ is the charge's position vector,

$$
\boldsymbol{g}=-\frac{M}{r^{2}} \hat{\boldsymbol{r}}
$$

is the Newtonian gravity of the central mass (with $r=|\boldsymbol{x}|$ and $\hat{\boldsymbol{r}}=\boldsymbol{x} / r)$, and

$$
\boldsymbol{f}_{\text {self }}=\lambda_{\mathrm{c}} \frac{q^{2} M}{r^{3}} \hat{\boldsymbol{r}}+\lambda_{\mathrm{rr}} \frac{2}{3} q^{2} \frac{d \boldsymbol{g}}{d t} \quad\left(\lambda_{\mathrm{c}}=\lambda_{\mathrm{rr}}=1\right)
$$

is the electromagnetic self-force. The first term on the right-hand side of Eq. (3) is the conservative part of the self-force; the second term is the usual expression for the electromagnetic radiation-reaction force. We work with units such that $G=c=1$, and we have inserted parameters $\lambda_{\mathrm{c}} \equiv 1, \lambda_{\mathrm{rr}} \equiv 1$ in order to later distinguish between conservative and radiation-reaction effects. In Appendix 
A we show that Mino's prescription [15] for the radiationreaction force gives rise precisely to the second term of Eq. (3). The adiabatic approximation to the electromagnetic self-force therefore consists of setting $\lambda_{\mathrm{c}}=0$ and $\lambda_{\mathrm{rr}}=1$ in Eq. (3).

Under the action of $\boldsymbol{g}$ alone the point charge would trace a Keplerian orbit of semilatus rectum $p$ and eccentricity $e$, and its orbital radius would be described by

$$
r=\frac{p}{1+e \cos (\phi-\omega)},
$$

where $\phi$ is the charge's true longitude and the constant $\omega$ is the longitude at periapsis. In Eq. (4) only $r$ and $\phi$ depend on time. The charge's energy per unit mass is $E=-M\left(1-e^{2}\right) /(2 p)$ and its angular momentum per unit mass is $L=\sqrt{M p}$. The orbital elements $(p, e)$ therefore act as a substitute for $(E, L)$ and can be adopted as the principal orbital elements. The remaining element $\omega$ is a positional orbital element. [A second positional element, $t_{0}$, does not appear explicitly in Eq. (4) and will not be needed in the sequel; it is defined by the statement $\phi\left(t_{0}\right)=\omega$.] Because the self-force of Eq. (3) keeps the orbital motion in a fixed plane, there is no need to introduce additional orbital elements.

Under the perturbation produced by the self-force the orbital motion is still described by Eq. (4) but the elements $(p, e, \omega)$ acquire a time dependence; the motion is tangent to an evolving osculating Keplerian orbit. Employing standard methods from celestial mechanics we show in Appendix B that the long-term evolution of the orbital parameters is governed by the differential equations

$$
\begin{aligned}
\langle\dot{p}\rangle & =-\lambda_{\mathrm{rr}} \frac{4}{3} \frac{q^{2} M}{m} \frac{\left(1-e^{2}\right)^{3 / 2}}{p^{2}}, \\
\langle\dot{e}\rangle & =-\lambda_{\mathrm{rr}} \frac{q^{2} M}{m} \frac{e\left(1-e^{2}\right)^{3 / 2}}{p^{3}}, \\
\langle\dot{\omega}\rangle & =-\lambda_{\mathrm{c}} \frac{1}{2} \frac{q^{2} M}{m} \sqrt{\frac{p}{M}} \frac{\left(1-e^{2}\right)^{3 / 2}}{p^{3}} .
\end{aligned}
$$

Here the overdot indicates a time derivative, and the angular brackets mean that $(\dot{p}, \dot{e}, \dot{\omega})$ are averaged over a complete period of the unperturbed orbit. It is assumed that $q^{2} \ll m p$ throughout the evolution, so that the time scales associated with the changes in $(p, e, \omega)$ are much longer than the orbital period.

Equations (5)-(7) describe the secular evolution of the orbital elements; they average out the unimportant oscillatory behavior that leads to a zero cumulative change over a large number of orbits. The decay of the principal elements $p$ and $e$ describes a shrinkage of the orbit accompanied by circularization. The presence of $\lambda_{\mathrm{rr}} \equiv 1$ in Eqs. (5) and (6) shows that these effects are purely dissipative, and that the evolution of $p$ and $e$ is driven entirely by the radiation-reaction part of the self-force. This evolution would be reproduced exactly by the adiabatic approximation. The negative sign on the righthand side of Eq. (7) indicates that the orbit undergoes

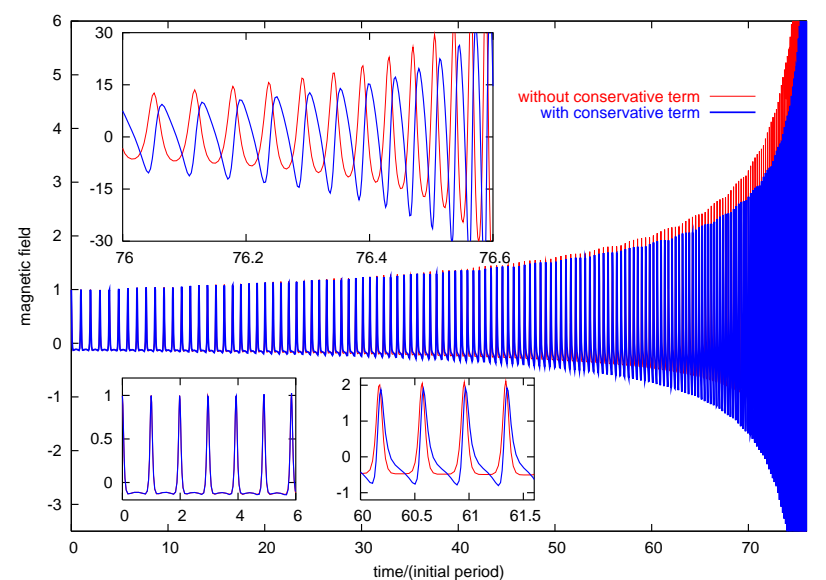

FIG. 1: Magnetic field produced by the orbiting particle, evaluated as a function of time in the wave zone, within the electric-dipole approximation. The units of the magnetic field are arbitrary, and the time is given in units of the initial orbital period. The initial orbital elements are $p_{0}=20 M$, $e_{0}=0.5$, and $\omega_{0}=0$. We set $q^{2} /\left(m p_{0}\right)=0.002$. In the color version of the figure, the blue curves represent the wave generated by an orbital evolution driven by the true self-force, and the red curves represent the wave calculated within the adiabatic approximation. In the black-and-white version the true wave appears thick and dark, while the approximate wave appears thin and light. The main plot shows the entire inspiral. The fact that the red/light curve appears to move upward relative to the blue/dark curve is a consequence of a relative phase shift driven by the secular evolution of $\omega$. The lower inset on the left shows the earliest part of the inspiral, before the phase shift had a chance to accumulate. The lower inset on the right shows a later portion of the inspiral, with the approximated wave (red/light) leading slightly in phase with respect to the true wave (blue/dark). Finally, the upper inset shows the latest portion of the inspiral, when the two waves are very much out of phase.

also a regression of its periapsis; the presence of $\lambda_{\mathrm{c}} \equiv 1$ shows that this effect is purely conservative. The evolution of $\omega$ is driven entirely by the conservative part of the self-force, and this evolution would not be reproduced by the adiabatic approximation.

We see that the conservative term in the electromagnetic self-force is indeed responsible for a secular effect, the accumulating regression of the orbit's periapsis. This effect would be missed altogether by the adiabatic approximation, which fails to account for the secular evolution of the positional orbital elements.

The secular evolution of $\omega$ has a direct effect upon the phasing of the orbit, and upon the phasing of the associated electromagnetic-wave signal. This statement is illustrated in Fig. 1, which displays the electromagnetic wave generated by the orbiting particle; the calculations behind this figure are presented in Appendix C. The figure shows very clearly that a model waveform calculated on the basis of the adiabatic approximation (which ignores the evolution of $\omega$ ) gradually goes out of phase with the true signal. This is in spite of the fact that both 
waves incorporate the same correct long-term evolution of $p$ and $e$.

The time scale associated with the dephasing can be estimated on the basis of Eq. (7). Assuming that $e$ is not too close to unity, this is given by $\langle\dot{\omega}\rangle \sim 1 / \tau_{\mathrm{dph}}$, or

$$
\tau_{\mathrm{dph}} \sim M\left(\frac{m M}{q^{2}}\right)\left(\frac{p}{M}\right)^{5 / 2} .
$$

The radiation-reaction time scale, on the other hand, can be estimated on the basis of Eq. (5). This is given by $\langle\dot{p}\rangle \sim p / \tau_{\mathrm{rr}}$, or

$$
\tau_{\mathrm{rr}} \sim M\left(\frac{m M}{q^{2}}\right)\left(\frac{p}{M}\right)^{3}
$$

The dephasing time is shorter than the radiation-reaction time by a factor of order $\sqrt{M / p} \ll 1$. The total phase shift $\Delta \Phi$ accumulated during a radiation-reaction time is estimated as $\Delta \Phi \sim\langle\dot{\omega}\rangle \tau_{\mathrm{rr}} \sim \tau_{\mathrm{rr}} / \tau_{\mathrm{dph}}$, or

$$
\Delta \Phi \sim \sqrt{p / M} \gg 1
$$

after using Eqs. (8) and (9). The accumulated phase shift is large, and this indicates that the adiabatic approach will not produce an adequate approximation of the true waveform over such a time interval.

\section{DISCUSSION}

Our two main conclusions are these:

- The secular evolution of the orbital elements is not driven only by the radiation-reaction part of the self-force; the conservative terms participate also. In particular, the conservative part of the self-force gives rise to a secular evolution of the positional elements.

- The secular evolution of the positional elements, which is not accounted for by an adiabatic approximation to the self-force, has measurable consequences. In particular, this evolution affects the phasing of the orbit and therefore the phasing of the associated wave.

These conclusions apply to the toy problem presented in the preceding section, but we firmly believe that they are not limited to this specific example. We are convinced that they hold in the context of the gravitational selfforce acting on a small body moving in the field of a Kerr black hole.

Returning to the gravitational problem, we emphasize that it should not come as a surprise that the conservative terms in the self-force can drive the secular evolution of the positional orbital elements. And it should not come as a surprise that this evolution can produce a measureable effect on the phasing of the gravitational waves. We shall elaborate on this observation in the next two paragraphs, but we note first that a similar point was made recently by Ajith et al. [27, 28].

Consider the post-Newtonian description of a twobody system, in a regime where $m$ (the small mass) is much smaller than $M$ (the large mass). The equations of motion for the relative orbit take the schematic form

$$
\boldsymbol{a}=\boldsymbol{g}_{0 \mathrm{PN}}+\boldsymbol{g}_{1 \mathrm{PN}}+\boldsymbol{g}_{2 \mathrm{PN}}+\boldsymbol{g}_{2.5 \mathrm{PN}}+\cdots,
$$

in which $\boldsymbol{a}$ is the acceleration of the relative position vector, $\boldsymbol{g}_{\text {OPN }}$ stands for the Newtonian gravitational field, and the additional terms are labeled by their postNewtonian order. Each term in the post-Newtonian expansion can be expanded in powers of $m / M$, so that

$$
\boldsymbol{g}_{\mathrm{nPN}}=\boldsymbol{g}_{\mathrm{nPN}}^{0}+\frac{m}{M} \boldsymbol{g}_{\mathrm{nPN}}^{1}+O\left(m^{2}\right) .
$$

Summation over post-Newtonian orders of the terms $\boldsymbol{g}_{\mathrm{nPN}}^{0}$ gives rise to an acceleration $\boldsymbol{a}^{0}$ which describes the geodesic motion of a test mass in the gravitational field of the mass $M$. Summation of the terms $(m / M) \boldsymbol{g}_{\mathrm{nPN}}^{1}$ gives rise to $(m / M) \boldsymbol{a}^{1}$, the correction to the geodesic motion that comes from the true self-force. These considerations show that in a post-Newtonian context, the self-force has conservative terms at order $0 \mathrm{PN}, 1 \mathrm{PN}, 2 \mathrm{PN}$, and higher, and it has dissipative terms at order 2.5PN and higher.

It is now easy to see why the conservative part of the self-force must drive a secular evolution of the positional elements. It is known, for example, that the test-mass term $\boldsymbol{g}_{1 \mathrm{PN}}^{0}$ drives a secular shift in $\omega$ relative to Keplerian motion; this is exemplified by the famous perihelion advance of Mercury. It is also known that $(m / M) \boldsymbol{g}_{1 \mathrm{PN}}^{1}$, the self-force term, contributes a correction of order $m / M$ to this shift [29]. Concretely, the post-Newtonian expression for the periapsis advance is given by Eq. (12.29) of Ref. 30],

$$
\langle\dot{\omega}\rangle_{1 \mathrm{PN}}=\frac{3\left(1-e^{2}\right)^{3 / 2}(M+m)^{3 / 2}}{p^{5 / 2}}
$$

since this depends on the total mass $M+m$, there is a test-mass contribution at order $m^{0}$ and a self-force contribution at order $m$. The self-force therefore produces a secular shift in $\omega$, over and above the effect already present in the test-mass limit. Because the conservative part of the self-force begins at a low post-Newtonian order, it should be clear that it will drive an evolution that is potentially more significant than the evolution driven by dissipative effects, which begin at order $2.5 \mathrm{PN}$. This property was featured in the toy model: The conservative part of the electromagnetic self-force can formally be thought of as a $1 \mathrm{PN}$ term in the equations of motion, while the radiation-reaction force is a smaller 1.5PN term. As we saw this led to a dephasing time that was shorter (by a factor of the orbital velocity) than the radiation-reaction time.

Incidentally, an extension of this argument shows why a recent claim made by Burko 31] must be erroneous. 
Burko claims that if the mass $m$ is spinning rapidly, then the effects of the conservative spin-orbit interaction will dominate over conservative self-force effects. It is easy to see why this claim must be false: Formally the spinorbit contribution to Eq. (11) begins at $1 \mathrm{PN}$ order, but for rapidly-spinning compact bodies the post-Newtonian scaling is in fact shifted to $1.5 \mathrm{PN}$ [32, 33]. Thus the spin-orbit interaction contributes a term $\boldsymbol{g}_{1.5 \mathrm{PN}}$, and the fact that the spin angular momentum scales as the mass squared implies that $\boldsymbol{g}_{\text {spin-orbit }}=(m / M) \boldsymbol{g}_{1.5 \mathrm{PN}}^{1}$. This addition to the self-force is therefore of $1.5 \mathrm{PN}$ order, and it will not dominate over the $1 \mathrm{PN}$ term that produces the periapsis shift.

These observations generalize to rapid motions and strong gravitational fields. In this context the conservative part of the self-force should still be expected to drive a secular evolution of the positional orbital elements those that characterize the initial position of the small body on the osculating geodesic. The secular evolution of the positional elements will affect the phasing of the orbit and the phasing of the associated gravitational wave. It is not, however, accounted for by the adiabatic approximation, which captures only the dissipative aspects of the orbital evolution.

We must add that there are important differences between weak-field and strong-field situations, and admit that our weak-field methods do not allow us to determine the strong-field effects associated with the conservative part of the self-force. We shall, nevertheless, attempt to draw some conclusions by extrapolating our weakfield results to strong-field situations. These conclusions, of course, are tentative and await confirmation from a proper strong-field investigation.

Our first observation is that in the case of rapid motions and strong fields, the conservative-driven evolution occurs over a time scale that is now comparable with (and not much shorter than) the radiation-reaction time scale. The separation of scales no longer occurs because the post-Newtonian scaling with the orbital velocity does not apply when the velocity is close to unity. This implies that the total accumulated phase shift now amounts to a number of wave cycles that may not be much larger than unity. Indeed, the estimate of Eq. (10) must be replaced by $\Delta \Phi \sim(p / M)^{3 / 2}$ in the case of gravity; this number would be large in a post-Newtonian situation, but in the present context we have $p / M \gtrsim 1$ and this leads to $\Delta \Phi \gtrsim 1$. This represents a fractional correction of order $m / M$ to the total number of wave cycles accumulated during the entire inspiral, which is of order $M / m$.

This strong-field estimate of the size of the accumulated phase shift is in agreement with statements made in Ref. 21] and in Sec. 1.2 of Drasco, Flanagan, and Hughes 20]. Recall the statement quoted earlier in Sec. II: "The effect of the dissipative pieces of the self force will accumulate secularly, while the effect of the conservative pieces will not. Hence the effect of the dissipative pieces on the phase of the orbit will be larger than that of the conservative pieces by a factor of the number of cycles of inspiral." While we still disagree with the first sentence, we acknowledge that the second sentence may well be true in the context of strong fields and rapid motions. But the fact that $\Delta \Phi$ scales as $(p / M)^{3 / 2}$ and must therefore be large in post-Newtonian situations seems to have been overlooked by these authors.

As a final remark we add that our considerations are guided by the realistic expectation that the orbital motion of a small compact body around a massive black hole will be eccentric and taking place outside the equatorial plane of the rotating black hole. For the special case of equatorial, circular orbits the relevance of the positional orbital elements disappears; the secular drift of $\omega$, for example, has no measurable consequences for circular orbits. In such special cases the adiabatic approximation can be expected to be reliable. We suspect that it is largely the consideration of circular, equatorial orbits that has boosted the confidence in this approach; refer, for example, to Appendix A of Drasco, Flanagan, and Hughes 20].

\section{Acknowledgments}

This work was supported by the Natural Sciences and Engineering Research Council of Canada. We have benefited from conversations with Leor Barack, Steve Detweiler, Bala Iyer, Eran Rosenthal, and Bernard Whiting. A correspondence with Steve Drasco, Eanna Flanagan, and Scott Hughes has been essential to the development of the conclusions presented in this paper.

\section{APPENDIX A: RETARDED, ADVANCED, AND RADIATION-REACTION FORCES}

The electromagnetic self-force acting on a point charge $q$ moving in an arbitrary curved spacetime was first calculated by DeWitt and Brehme 25], and their expression was later corrected by Hobbs 34]. We briefly sketch the main steps of the derivation here, relying heavily on the presentation given in Sec. 5.2 of Ref. [4] (hereafter referred to as LRR). We then provide an expression for the advanced version of the self-force. Subtracting the two gives Mino's radiation-reaction force [15]. Finally, we evaluate these forces in the case of a charge moving slowly in the weak gravity of a central mass $M$, relying heavily on the work of DeWitt and DeWitt 24] as reviewed in Ref. [26] (hereafter referred to as PP).

The derivation of the true, retarded, self-force begins with the following expression for the retarded potential produced by a charged particle moving on a world line described by the parametric relations $z^{\mu}(\tau)$ [LLR Sec. 5.2.2, Eq. (443)]:

$$
A_{\mathrm{ret}}^{\alpha}(x)=q \int G_{\mathrm{ret} \mu}^{\alpha}(x, z(\tau)) u^{\mu}(\tau) d \tau .
$$


Here $x$ is the spacetime point at which the potential is evaluated, $G_{\mathrm{ret} \alpha^{\prime}}^{\alpha}\left(x, x^{\prime}\right)$ is the retarded Green's function associated with the wave equation satisfied by $A^{\alpha}$ (in the Lorenz gauge), and $u^{\mu}=d z^{\mu} / d \tau$ is the charge's velocity vector.

The electromagnetic field $F_{\text {ret }}^{\alpha \beta}(x)$ obtained from the retarded potential is singular on the world line, and it must be regularized before taking the limit $x \rightarrow z$ and evaluating the Lorentz force $q F_{\nu}^{\mu}(z) u^{\nu}$. Detweiler and Whiting 9] have shown that the correct regularization procedure is to remove from $F_{\text {ret }}^{\alpha \beta}(x)$ a singular field $F_{\mathrm{S}}^{\alpha \beta}(x)$ which is known to exert no force on the particle. The singular field is defined and evaluated in Sec. 5.2.5 of LRR, and the regular remainder

$$
F_{\mathrm{R}}^{\alpha \beta}(x)=F_{\text {ret }}^{\alpha \beta}(x)-F_{\mathrm{S}}^{\alpha \beta}(x)
$$

is what must be substituted into the Lorentz-force equation.

When the charged particle moves freely (being subjected to no other force but its own self-force) in a vacuum region of the spacetime, the resulting equations of motion are $m a^{\mu}=f_{\text {ret }}^{\mu}$, where $m$ is the particle's mass, $a^{\mu}$ its covariant acceleration, and [LRR Sec. 2.5.6, Eq. (481)]

$$
f_{\mathrm{ret}}^{\mu}(\tau)=2 q^{2} u_{\nu} \int_{-\infty}^{\tau^{-}} \nabla^{[\mu} G_{\operatorname{ret} \lambda^{\prime}}^{\nu]}\left(z(\tau), z\left(\tau^{\prime}\right)\right) u^{\lambda^{\prime}}\left(\tau^{\prime}\right) d \tau^{\prime}
$$

is the true, retarded, electromagnetic self-force. Notice that the integration extends over the particle's past history, and that it is cut short at $\tau^{\prime}=\tau^{-}=\tau-0^{+}$to avoid the singular behavior of the retarded Green's function at coincidence. This limiting procedure was first derived by DeWitt and Brehme [25], and it is a natural byproduct of the Detweiler-Whiting regularization method 9 ].

The advanced version of the self-force is obtained by starting with the advanced solution for the potential,

$$
A_{\text {adv }}^{\alpha}(x)=q \int G_{\operatorname{adv} \mu}^{\alpha}(x, z(\tau)) u^{\mu}(\tau) d \tau,
$$

and going through the same calculational steps as described above. The advanced electromagnetic field $F_{\text {adv }}^{\alpha \beta}(x)$ also is singular on the world line, but it is regularized by the same singular field $F_{\mathrm{S}}^{\alpha \beta}(x)$ as the retarded field. The end result for the equations of motion is $m a^{\mu}=f_{\mathrm{adv}}^{\mu}$, with

$$
f_{\mathrm{adv}}^{\mu}(\tau)=2 q^{2} u_{\nu} \int_{\tau^{+}}^{\infty} \nabla^{[\mu} G_{\mathrm{adv} \lambda^{\prime}}^{\nu]}\left(z(\tau), z\left(\tau^{\prime}\right)\right) u^{\lambda^{\prime}}\left(\tau^{\prime}\right) d \tau^{\prime}
$$

being the advanced version of the electromagnetic selfforce. Notice that the integration now extends over the particle's future history, and that it is cut short at $\tau^{\prime}=\tau^{+}=\tau+0^{+}$to avoid the singular behavior of the advanced Green's function at coincidence.

Mino's radiation-reaction force is defined by 15]

$$
f_{\mathrm{rr}}^{\mu}=\frac{1}{2}\left(f_{\mathrm{ret}}^{\mu}-f_{\mathrm{adv}}^{\mu}\right) .
$$

This depends on the particle's entire history from $\tau^{\prime}=$ $-\infty$ to $\tau^{\prime}=+\infty$. Because the retarded and advanced fields are equally singular on the world line, the evaluation of the radiation-reaction force does not require regularization. As was mentioned in Sec. II of the main text, the total work done by the radiation-reaction force equals the total energy radiated by the charged particle [18.

The expressions (A3), A5 , and (A6) for the retarded, advanced, and radiation-reaction forces are valid for any charge that moves freely in a vacuum region of an arbitrary spacetime. Let us now specialize to the case of a particle moving slowly in the weakly curved spacetime of a (noncompact) star of mass $M$. The steps required to compute the retarded Green's function for such a spacetime, and to evaluate the integral of Eq. (A3), are detailed in PP [26]. They lead to [PP Eq. (5.23)]

$$
\boldsymbol{f}_{\mathrm{ret}}=\frac{q^{2} M}{r^{3}} \hat{\boldsymbol{r}}+\frac{2}{3} q^{2} \frac{d \boldsymbol{g}}{d t},
$$

which is also Eq. (3) of Sec. IV, where the notation is introduced. Going through the same steps, starting instead with Eq. A5), gives

$$
\boldsymbol{f}_{\mathrm{adv}}=\frac{q^{2} M}{r^{3}} \hat{\boldsymbol{r}}-\frac{2}{3} q^{2} \frac{d \boldsymbol{g}}{d t} .
$$

Not surprisingly, the advanced self-force is obtained from the retarded self-force by time reversal, $t \rightarrow-t$. Finally, from Eqs. A6 - A8 we obtain Mino's radiation-reaction force,

$$
\boldsymbol{f}_{\mathrm{rr}}=\frac{2}{3} q^{2} \frac{d \boldsymbol{g}}{d t} .
$$

This is the familiar result that follows from the Abrahams-Lorentz-Dirac equation in flat spacetime (see, for example, Ch. 16 of Ref. [35]).

\section{APPENDIX B: ORBITAL EVOLUTION UNDER THE SELF-FORCE}

In this Appendix we provide a derivation of Eqs. (5) (17). We rely on standard analytical methods of celestial mechanics as described, for example, in Ref. [36].

We work in the $x-y$ plane and introduce the polar coordinates $(r, \phi)$ defined by $x=r \cos \phi$ and $y=r \sin \phi$. We use the basis $(\hat{\boldsymbol{r}}, \hat{\boldsymbol{\phi}})$, in which $\hat{\boldsymbol{r}}$ is a unit vector pointing in the direction of increasing $r$, and $\hat{\phi}$ is a unit vector pointing in the direction of increasing $\phi$. The particle's velocity vector, for example, can be decomposed as $\boldsymbol{v}=\dot{\boldsymbol{r}} \hat{\boldsymbol{r}}+(r \dot{\phi}) \hat{\boldsymbol{\phi}}$, where a dot indicates differentiation with respect to $t$.

The self-force of Eq. (3) admits the decomposition

$$
\boldsymbol{f}_{\mathrm{self}}=m(R \hat{\boldsymbol{r}}+S \hat{\boldsymbol{\phi}}),
$$


with

$$
R=\frac{q^{2} M}{m}\left(\lambda_{\mathrm{c}} \frac{1}{r^{3}}+\lambda_{\mathrm{rr}} \frac{4}{3} \frac{\dot{r}}{r^{3}}\right)
$$

and

$$
S=\frac{q^{2} M}{m}\left(-\lambda_{\mathrm{rr}} \frac{2}{3} \frac{\dot{\phi}}{r^{2}}\right) .
$$

We treat $q^{2} / m$ as a small parameter and the self-force as a small perturbing force that produces only a slight deviation with respect to Keplerian motion.

The charge's Keplerian orbit is described by Eq. (4), which we rewrite as

$$
r=\frac{p}{1+e \cos v}
$$

in terms of the true anomaly $v$; the longitude is then given by $\phi=v+\omega$. For Keplerian motion the orbital elements $(p, e, \omega)$ are constant, and $t(v)$ is determined by integrating the differential equation

$$
\dot{v}=\sqrt{\frac{M}{p^{3}}}(1+e \cos v)^{2} .
$$

Combining Eqs. (B4) and (B5) produces

$$
\dot{r}=e \sqrt{\frac{M}{p}} \sin v .
$$

The orbital period is the time required by the particle to go from periapsis $(v=0)$ to apoapsis $(v=\pi)$ and then back to periapsis $(v=2 \pi)$. It is given by

$$
P=2 \pi \sqrt{\frac{p^{3}}{\left(1-e^{2}\right)^{3} M}} .
$$

During this interval $\phi$ increases from $\omega$ to $\omega+2 \pi$.

The electromagnetic self-force perturbs the particle's motion away from its Keplerian orbit. In the method of osculating orbital elements the perturbed motion is still described exactly by Eqs. (B4)-(B6), but the elements $(p, e, \omega)$ acquire a time dependence. The driving equations are (see, for example, Ch. 11 of Ref. [36])

$$
\begin{aligned}
\dot{p} & =2 \sqrt{\frac{p^{3}}{M}} \frac{S}{1+e \cos v}, \\
\dot{e} & =\sqrt{\frac{p}{M}}\left[R \sin v+S \frac{2 \cos v+e\left(1+\cos ^{2} v\right)}{1+e \cos v}\right], \\
\dot{\omega} & =\sqrt{\frac{p}{M}} \frac{1}{e}\left[-R \cos v+S \frac{(2+e \cos v) \sin v}{1+e \cos v}\right],
\end{aligned}
$$

where $R$ and $S$ are listed in Eqs. (B2) and (B3), respectively.

For the purpose of obtaining the secular behavior of the orbital elements, it is sufficient to integrate Eqs. (B8)(B10) approximately, employing the method of averaging (see, for example, Ch. 12 of Ref. [37]). In this method we average the right-hand side of each equation over a complete orbital period, treating $(p, e, \omega)$ as constants within the integral. If, for example, $\dot{I}(p, e, \omega ; v)$ denotes the time derivative of orbital element $I$, then we calculate

$$
\langle\dot{I}\rangle \equiv \frac{1}{P} \int_{0}^{P} \dot{I}(p, e, \omega ; v) d t=\frac{1}{P} \int_{0}^{2 \pi} \frac{\dot{I}(p, e, \omega ; v)}{\dot{v}} d v,
$$

using Eq. (B5) for $\dot{v}$ and keeping $(p, e, \omega)$ fixed while integrating. The result discards the rapid oscillations in $I(t)$ which do not accumulate over a large number of orbits. It keeps, however, the secular drift that eventually produces a large cumulative change.

Substitution of Eqs. (B8) (B10) into the integral of Eq. (B11) leads to the results displayed in Eqs. (5)-(7). We recall that only the radiation-reaction component of the self-force is responsible for the secular evolution of $p$ and $e$, while only the conservative component is responsible for the secular change in $\omega$.

We note that in the case of a purely conservative selfforce [obtained by setting $\lambda_{\mathrm{rr}}=0$ in Eq. (3)] the relation between $\phi$ and $v$ can be obtained exactly: $\phi(v)=[1-$ $\left.q^{2} /(m p)\right]^{1 / 2} v$. This exact expression leads to a modified form of Eq. (7),

$$
\langle\dot{\omega}\rangle=-\frac{1}{2} \frac{q^{2} M}{m} \sqrt{\frac{p}{M}} \frac{\left(1-e^{2}\right)^{3 / 2}}{p^{3}} f(x),
$$

where $x \equiv q^{2} /(m p)$ and

$$
f(x)=\frac{1-\sqrt{1-x}}{x / 2}=1+\frac{x}{4}+\frac{x^{2}}{8}+\cdots .
$$

When $q^{2} \ll m p$ we have that $f(x) \simeq 1$ and Eq. (7) is a very good approximation to Eq. (B12).

In Appendix $\mathrm{C}$ we will need explicit expressions for $\langle p\rangle(v),\langle e\rangle(v)$, and $\langle\omega\rangle(v)$. To obtain these we first average $I^{\prime} \equiv d I / d v=\dot{I} / \dot{v}$ over a complete orbital period, to get $\left\langle I^{\prime}\right\rangle \equiv(2 \pi)^{-1} \int_{0}^{2 \pi} I^{\prime} d v=(P / 2 \pi)\langle\dot{I}\rangle$. This yields

$$
\begin{aligned}
\left\langle p^{\prime}\right\rangle & =-\lambda_{\mathrm{rr}} \frac{4}{3} \frac{q^{2}}{m} \sqrt{\frac{M}{p}}, \\
\left\langle e^{\prime}\right\rangle & =-\lambda_{\mathrm{rr}} \frac{q^{2}}{m} e \sqrt{\frac{M}{p^{3}}} \\
\left\langle\omega^{\prime}\right\rangle & =-\lambda_{\mathrm{c}} \frac{1}{2} \frac{q^{2}}{m} \frac{1}{p} .
\end{aligned}
$$

Next we integrate, and obtain

$$
\begin{aligned}
\langle p\rangle & =p_{0}\left(1-\lambda_{\mathrm{rr}} v / v_{0}\right)^{2 / 3}, \\
\langle e\rangle & =e_{0}\left(1-\lambda_{\mathrm{rr}} v / v_{0}\right)^{1 / 2}, \\
\langle\omega\rangle & =-\frac{3}{4} \sqrt{\frac{p_{0}}{M}}\left[1-\left(1-\lambda_{\mathrm{c}} v / v_{0}\right)^{1 / 3}\right],
\end{aligned}
$$


where

$$
v_{0}=\frac{m}{2 q^{2}} \sqrt{\frac{p_{0}^{3}}{M}} .
$$

We assume that the orbital evolution starts at $v=0$ with values $\left(p=p_{0}, e=e_{0}, \omega=0\right)$ for the orbital elements. The evolution ends at $v=v_{0}$ when both $\langle p\rangle$ and $\langle e\rangle$ are zero; the final value of the periapsis shift is $\langle\omega\rangle\left(v_{0}\right)=-(3 / 4) \sqrt{p_{0} / M}$, and this expression confirms the estimate of Eq. (10).

\section{APPENDIX C: ELECTROMAGNETIC WAVE}

We calculate the electromagnetic wave (or more precisely, the wave-zone magnetic field) produced by a charged particle subjected to the electromagnetic selfforce of Eq. (3). As was explained in Appendix B, the motion is at all times described by $r(v)$ given by Eq. (B4), $\phi(v)=v+\omega$, and $t(v)$ is obtained by numerically integrating Eq. (B5). The orbital elements $(p, e, \omega)$ evolve according to Eqs. B17 B20.

In the electric-dipole approximation the wave-zone magnetic field is given by (see, for example, Sec. 11.1.4 of Ref. [38])

$$
\boldsymbol{B}=-\frac{\mu_{0}}{4 \pi} \frac{\hat{\boldsymbol{n}} \times \ddot{\boldsymbol{p}}}{c d}
$$

where $\hat{\boldsymbol{n}}=[\sin \alpha \cos \beta, \sin \alpha \sin \beta, \cos \alpha]$ is a unit vector which points from the source to the detector, and $\boldsymbol{p}$ is the source's electric dipole moment expressed in terms of retarded time $t-d / c$; the detector is at a distance $d$ from the source and $c$ is the speed of light. For an orbiting charge we have $\ddot{\boldsymbol{p}}=q \boldsymbol{a}$, where $\boldsymbol{a}$ is the particle's acceleration. For our purposes it is sufficient to use the approximation $\boldsymbol{a}=\boldsymbol{g}=-\left(M / r^{2}\right) \hat{\boldsymbol{r}}$ and Eq. (C1) becomes

$$
\boldsymbol{B}=\frac{\mu_{0}}{4 \pi} \frac{q M}{c d} \frac{\hat{\boldsymbol{n}} \times \hat{\boldsymbol{r}}}{r^{2}} .
$$

With $\hat{\boldsymbol{r}}=[\cos \phi, \sin \phi, 0]$ we have

$$
\hat{\boldsymbol{n}} \times \hat{\boldsymbol{r}}=[-\cos \alpha \sin \phi, \cos \alpha \cos \phi, \sin \alpha \sin (\phi-\beta)],
$$

and each component of the magnetic field can be computed straightforwardly.

To produce the plots of Fig. 1 we chose the viewing angles $(\alpha=\pi / 4, \beta=0)$ and selected the $y$ component of the magnetic field. To integrate the equations of motion we set $p_{0}=20 M, e_{0}=0.5$, and $q^{2} /\left(m p_{0}\right)=0.002$. The magnetic field was rescaled by an arbitrary numerical factor to obtain values of order unity. To produce the true wave (represented as a blue/dark curve) we set $\lambda_{\text {rr }}=$ $\lambda_{\mathrm{c}}=1$ in Eqs. B17 B19). To produce the adiabatic approximation to the wave (represented as a red/light curve) we selected $\lambda_{\mathrm{rr}}=1$ and $\lambda_{\mathrm{c}}=0$.
[1] The LISA web site is located at http://lisa.jpl.nasa.gov/.

[2] Y. Mino, M. Sasaki, and T. Tanaka, Phys. Rev. D 55, 3457 (1997), arXiv:gr-qc/9712056.

[3] T. C. Quinn and R. M. Wald, Phys. Rev. D 56, 3381 (1997), arXiv:gr-qc/9610053.

[4] E. Poisson, Living Rev. Relativity 7 (2004), 6. [Online article]: cited on June 19, 2021, http://www.livingreviews.org/lrr-2004-6.

[5] P. L. Chrzanowski, Phys. Rev. D 11, 2042 (1975).

[6] A. Ori, Phys. Rev. D 67, 124010 (2003), arXiv:grqc/0207045.

[7] C. O. Lousto and B. F. Whiting, Phys. Rev. D 66, 024026 (2002), arXiv:gr-qc/0203061.

[8] S. A. Teukolsky, Astrophys. J. 185, 635 (1973).

[9] S. Detweiler and B. F. Whiting, Phys. Rev. D 67, 024025 (2003), arXiv:gr-qc/0202086.

[10] L. Barack, Y. Mino, H. Nakano, A. Ori, and M. Sasaki, Phys. Rev. Lett. 88, 091101 (2002), arXiv:gr-qc/0111001.

[11] L. Barack and A. Ori, Phys. Rev. D 67, 024029 (2003), arXiv:gr-qc/0209072.

[12] L. Barack and A. Ori, Phys. Rev. Lett. 90, 111101 (2003), arXiv:gr-qc/0212103.

[13] Y. Mino, H. Nakano, and M. Sasaki, Prog. Theor. Phys. 108, 1039 (2002), arXiv:gr-qc/0111074.

[14] L. Barack and A. Ori, Phys. Rev. D 64, 124003 (2001), arXiv:gr-qc/0107056.

[15] Y. Mino, Phys. Rev. D 67, 084027 (2003), arXiv:grqc/0302075.

[16] Y. Mino, Prog. Theor. Phys. 113, 733 (2005), arXiv:gr- qc/0506003.

[17] Y. Mino, Class. Quantum Grav. 22, S717 (2005), arXiv:gr-qc/0506002.

[18] T. C. Quinn and R. M. Wald, Phys. Rev. D 60, 064009 (1999), arXiv:gr-qc/9903014.

[19] D. V. Gal'tsov, J. Math. Phys. A: Math. Gen. 15, 3737 (1982).

[20] S. Drasco, E. E. Flanagan, and S. A. Hughes, Class. Quantum Grav. 22, S801 (2005), arXiv:gr-qc/0505075.

[21] S. A. Hughes, S. Drasco, E. E. Flanagan, and J. Franklin, Phys. Rev. Lett. 94, 221101 (2005), arXiv:gr-qc/0504015.

[22] N. Sago, T. Tanaka, W. Hikida, and H. Nakano, Prog. Theor. Phys. 114, 509 (2005), arXiv:gr-qc/0506092.

[23] S. Drasco and S. A. Hughes, Gravitational wave snapshots of generic extreme mass ratio inspirals (2005), arXiv:gr-qc/0509101.

[24] C. Morette DeWitt and B. S. DeWitt, Physics (Long Island City, N.Y.) 1, 3 (1964).

[25] B. S. DeWitt and R. W. Brehme, Ann. Phys. (N.Y.) 9, 220 (1960).

[26] M. J. Pfenning and E. Poisson, Phys. Rev. D 65, 084001 (2002), arXiv:gr-qc/0012057.

[27] P. Ajith, B. R. Iyer, C. A. K. Robinson, and B. S. Sathyaprakash, Phys. Rev. D 71, 044029 (2005), erratum-ibid. D 72, 049902 (2005), arXiv:gr-qc/0412033.

[28] P. Ajith, B. R. Iyer, C. A. K. Robinson, and B. S. Sathyaprakash, Class. Quantum Grav. 22, S1179 (2005), arXiv:gr-qc/0503124.

[29] T. Damour and N. Deruelle, Ann. Inst. Henri Poincare 
44, 263 (1986)

[30] C. M. Will, Theory and Experiment in Gravitational Physics, Revised Edition (Cambridge University Press, Cambridge, England, 1993).

[31] L. M. Burko, Phys. Rev. D 69, 044011 (2004), arXiv:grqc/0308003.

[32] L. E. Kidder, C. M. Will, and A. G. Wiseman, Phys. Rev. D 47, R4183 (1993).

[33] L. E. Kidder, Phys. Rev. D 52, 821 (1995).

[34] J. M. Hobbs, Ann. Phys. (N.Y.) 47, 141 (1968).
[35] J. D. Jackson, Classical Electrodynamics, Third Edition (Wiley, New York, 1999).

[36] J. M. A. Danby, Fundamentals of celestial mechanics. Second edition (Willmann-Bell, Richmond, 1988).

[37] P. B. Kahn, Mathematical methods for scientists and engineers. Linear and nonlinear systems (Dover, Mineola, 1990).

[38] D. J. Griffiths, Introduction to electrodynamics. Third edition (Prentice Hall, Upper Saddle River, 1999). 